\title{
Difference between cerebral embolic events following Transcatheter Aortic Valve Implantation (TAVI) and Surgical Aortic Valve Replacement (SAVR): a diffusion weighted MRI study
}

\author{
Akhlaque Uddin ${ }^{*}$, Timothy Fairbairn ${ }^{1}$, Ibrahim Djoukhader ${ }^{2}$, Stuart Currie ${ }^{2}$, Christopher D Steadman ${ }^{3}$, \\ Manish Motwani ${ }^{1}$, Ananth Kidambi ${ }^{1}$, Anthony Goddard ${ }^{2}$, Daniel Blackman ${ }^{4}$, Gerry P McCann ${ }^{3}$, Sven Plein ${ }^{1}$, \\ John P Greenwood ${ }^{1}$
}

From 16th Annual SCMR Scientific Sessions

San Francisco, CA, USA. 31 January - 3 February 2013

\section{Background}

Transcatheter Aortic Valve Implantation (TAVI) is used to treat symptomatic severe aortic stenosis in a non-surgical high risk population. The incidence of stroke and micro-infarction is higher in the TAVI population compared to surgical aortic valve replacement (SAVR) at 30 days, which may be due to various factors such as valve calcification and aortic atheroma. However, the natural history and clinical consequences of micro-infarction is unknown.

\section{Methods}

Cerebral imaging was conducted before TAVI/SAVR, at $<7$ days post-procedure and 6 months post-procedure. MRI scans were performed on a $1.5 \mathrm{~T}$ system (Intera, Philips or Avanto, Siemens) using a protocol of T2 weighted fast field echo, T2 turbo field echo and diffusion weighted imaging (DWI) (22 slices, $5 \mathrm{~mm}$ thick, $1 \mathrm{~mm}$ gap, FOV 350, RFOV 100). Three neuroradiologists independently analysed the scans and were blinded o clinical details to avoid bias. New cerebral lesions were measured $(<5 \mathrm{~mm}$ or $>5 \mathrm{~mm})$ and the vascular territory described. Quantification of cerebral infarct lesion volume was performed by manual planimetry using post processing software (Qmass 7.2 Medis, The Netherlands).

\footnotetext{
${ }^{1}$ Academic Unit of Cardiovascular Medicine, Multidisciplinary Cardiovascular Research Centre (MCRC) \& Leeds Institute of Genetics, Health and Therapeutics, Leeds, UK

Full list of author information is available at the end of the article
}

\section{Results}

45 TAVI and 21 SAVR patients were studied. Mean age for TAVI was $80 \pm 5.9$ years, for SAVR $69 \pm 8.8$ years: Logistic EuroSCORE for TAVI was $19.1 \pm 13$, for SAVR 7.1 \pm 2.7 . In the TAVI patients $82 \%$ had new embolic lesions on DWI (Figure 1) compared to $47.6 \%$ of the SAVR group. Two TAVI patients had a clinical stroke with none in the SAVR group. There was a higher cerebral infract lesion volume in TAVI group compared to SAVR group $(1.74 \pm 2.8 \mathrm{ml}$ vs. $0.41 \pm 0.48 \mathrm{ml} \mathrm{p}=0.01)$. At 6 months $17 \mathrm{TAVI}$ patients $(11$ were unable to attend, 10 had died, 7 had a pacemaker)

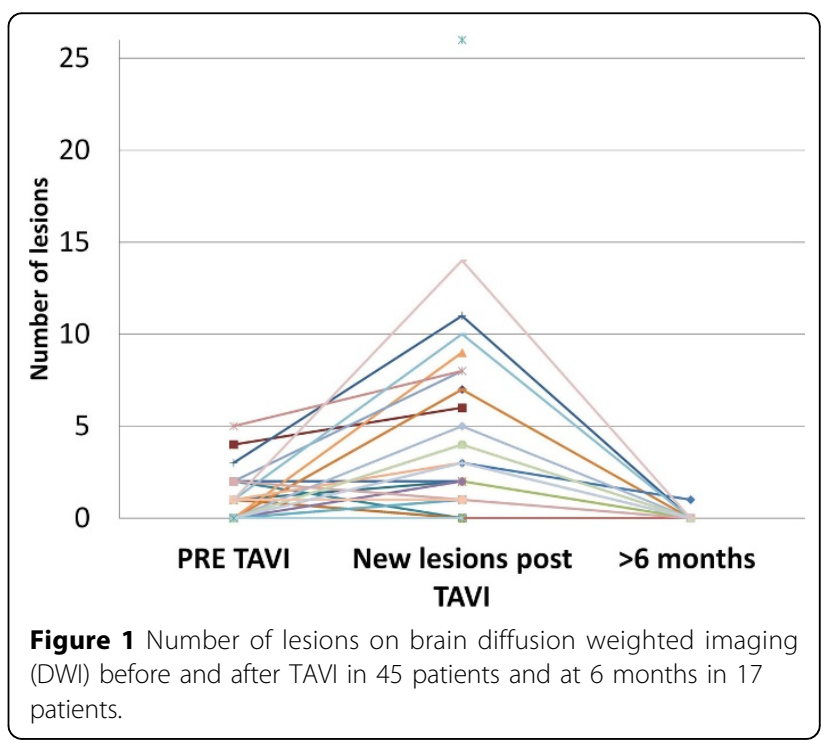




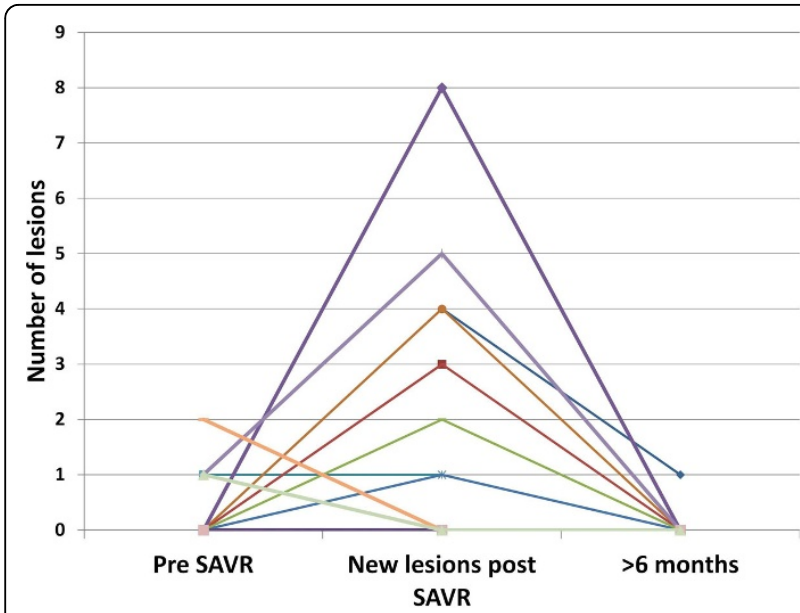

Figure 2 Number of lesions on brain diffusion weighted imaging (DWI) before and after surgical aortic valve replacement(SAVR) in 21 patients and at 6 months in 18 patients.

and 18 SAVR (1 died and 1 was unable to attend) were rescanned. Only 1 TAVI and 1 SAVR patient had a new subclinical micro-infarct. All previously detected microinfarcts had completely resolved.

\section{Conclusions}

There is a significantly greater number of new microembolic events after TAVI compared to SAVR. However all of these lesions appear to completely resolve by 6 months.

\section{Funding}

SP is funded by a British Heart Foundation fellowship (FS/10/62/28409).

SP and JPG receive an educational research grant from Philips Healthcare.

\section{Author details \\ ${ }^{1}$ Academic Unit of Cardiovascular Medicine, Multidisciplinary Cardiovascular Research Centre (MCRC) \& Leeds Institute of Genetics, Health and Therapeutics, Leeds, UK. ²Department of Neuroradiology, Leeds Teaching Hospitals NHS trust, Leeds General Infirmary, Leeds, UK. ${ }^{3}$ National Institute for Health Research(NIHR) Leicester Cardiovascular Biomedical Research Unit, University of Leicester, Leicester, UK. ${ }^{4}$ Department of Cardiology, Leeds Teaching Hospitals NHS trust, Leeds General Infirmary, Leeds, UK.}

Published: 30 January 2013

\section{doi:10.1186/1532-429X-15-S1-059}

Cite this article as: Uddin et al:: Difference between cerebral embolic events following Transcatheter Aortic Valve Implantation (TAVI) and Surgical Aortic Valve Replacement (SAVR): a diffusion weighted MRI study. Journal of Cardiovascular Magnetic Resonance 2013 15(Suppl 1):O59.

\section{Submit your next manuscript to BioMed Central} and take full advantage of:

- Convenient online submission

- Thorough peer review

- No space constraints or color figure charges

- Immediate publication on acceptance

- Inclusion in PubMed, CAS, Scopus and Google Scholar

- Research which is freely available for redistribution

Submit your manuscript at www.biomedcentral.com/submit 